\title{
Overlapping memory replay during sleep builds cognitive schemata
}

\author{
Penelope A. Lewis and Simon J. Durrant
}

School of Psychological Sciences, University of Manchester, Zochonis Building, Brunswick Street, Manchester M13 9PL, UK

\begin{abstract}
Sleep enhances integration across multiple stimuli, abstraction of general rules, insight into hidden solutions and false memory formation. Newly learned information is better assimilated if compatible with an existing cognitive framework or schema. This article proposes a mechanism by which the reactivation of newly learned memories during sleep could actively underpin both schema formation and the addition of new knowledge to existing schemata. Under this model, the overlapping replay of related memories selectively strengthens shared elements. Repeated reactivation of memories in different combinations progressively builds schematic representations of the relationships between stimuli. We argue that this selective strengthening forms the basis of cognitive abstraction, and explain how it facilitates insight and false memory formation.
\end{abstract}

\section{What's the gist?}

I do not remember many details about childhood birthday parties. There was one when we played blind man's bluff, and another with cucumber sandwiches, but in general these affairs blur together under the broad heading of birthdays. About this category I have much information: I know the parties involved hordes of children, that there were presents and invariably a cake. However, which birthday belonged to whom, and exactly what was done there escapes me. This is unsurprising as chronologically old memories rarely include clear episodic details but these aspects of a situation or memory type that are repeated again and again are normally recalled with ease. Central shared concepts of this type can be thought of as the 'gist' (see Glossary), or essential features, of a set of memories, and taken together can form a cognitive framework of expectations or schema (Box 1). This article proposes a physiological mechanism by which memory replay during sleep could be responsible for the abstraction of gist information from newly encoded memories to form cognitive schemata.

Recent research has shown that sleep facilitates abstraction of gist information [1-5] as well as integration across multiple memories [6-8], insight into hidden solutions [9], and even the ability to make creative connections between distantly related ideas and concepts [10]. These findings build upon a strong body of work demonstrating that sleep plays a crucial role in memory consolidation (for reviews [11-13]), as well as a highly influential model of how memories are re-organised and strengthened by

Corresponding author: Lewis, P.A. (P.Lewis@Manchester.ac.uk). replay during slow wave sleep (SWS) (see $[11,12,14-17]$ and Box 2). This model elegantly explains how the specific physiology of SWS, in combination with the replay of memories that has been observed in both rats and humans during this sleep stage (Box 3), could assist consolidation by transferring memories from a short-term store in the hippocampus to longer term cortical representations. Importantly, however, this model cannot presently explain why sleep is beneficial for gist extraction, insight or the formation of false memories.

A separate line of research investigating the formation of conceptual frameworks has shown that newly learned 


\section{Box 1. Schema theory}

A schema is a cognitive structure that represents organised knowledge of some part of the world that is acquired on the basis of experience [52-54]. The concept was initially proposed by Piaget in specific reference to the development of children's motor programs [55], and subsequently developed by Bartlett [56] and later by cognitive psychologists such as Mandler [54] and Howard [52], who expanded the meaning to include conceptual knowledge.

Different types of schemata have been proposed, including motor schemata [57], event schemata, scene schemata and story schemata [54]. Although these differ in many details, they share common defining properties and processes. Schemata are 'initially formed' through the implicit abstraction of regularities within the environment, together with the discarding of differences. A dog schema, for example, could come about through the recognition that all dogs bark and have four legs. Dogs, however, come in different colours and sizes, and so a general dog schema would leave these slots open. An existing schema is 'activated' when a triggering event or object is encountered, such as seeing a dog or being told that a friend is bringing a dog. The 'triggered' schema includes a set of expectations. If my friend's dog barks and has four legs, then the memory of this dog will be 'assimilated' in my dog schema. This process could result in atypical features of the dog being forgotten or replaced in my memory with a more typical characteristic. If instead my friend's dog has only three legs, and I also regularly encounter other friends whose dogs have three legs, I might be forced to revise my schema to 'accommodate' this new perspective. Assimilation and accommodation represent the two main alternatives in the relation between new memories and schemata.

An intriguing recent study into schema formation in rats revealed that an environment schema assimilating information about placeflavour associations required several weeks to become fully formed [18]. Once complete, however, this knowledge became independent of the hippocampus. Importantly, once the rats developed this schema, new place-flavour associations could be learned much more rapidly, presumably because they now had a cognitive framework in which to store this knowledge.

Where might schemata reside in humans if independent of the hippocampus? van Kesteren and colleagues [19] manipulated the formation of a movie schema by scrambling the order of events in the first part of the movie for some participants (scrambled group) while maintaining the original order for others (ordered group). Unsurprisingly, the ordered group scored better when schematic knowledge of the first part of the movie was tested, whereas there was no betweengroup difference in nonschematic memory. Notably, functional connectivity between the hippocampus and the ventromedial prefrontal cortex (vmPFC) was stronger in the scrambled group than the ordered group while watching the second (unscrambled) part of the movie. This suggests that these structures might interact during schema formation, and therefore this finding supports a role for the vmPFC in schema formation and/or in the representation of partially consolidated schemata. information is more rapidly assimilated if it is compatible with an existing set of ideas or 'schema'. Building on this, recent work suggests that information is more rapidly transferred from the hippocampus to the neocortex in the presence of such a schema [18-20], but the mechanism for this facilitation and sleep's potential role in this remain to be understood.

Here, we attempt to integrate these two lines of investigation by describing a physiologically plausible mechanism through which the replay of new memories during SWS could underpin both schema formation [18] and the integration of new knowledge into existing frameworks $[7,8]$. This mechanism is compelling in its simplicity and in its effortless fit to the literature on reactivation of memories in rats [21,22] and humans [23-26], as well as to research on concept formation [27]. It explains why newly learned material becomes increasingly easy to integrate and remember as a conceptual schema takes shape $[18,19]$. It also explains the observed postsleep enhancements in integration [6-8], abstraction [1-5], insight [9] and false memory formation [28].

\section{Overlapping replay and schema formation}

Offline memory replay is frequent, widespread and strongly associated with subsequent strengthening of memories (Box 3). This section will explain how the overlapping replay of multiple memories during SWS could serve to strengthen areas of commonality (Figure 1). It will also

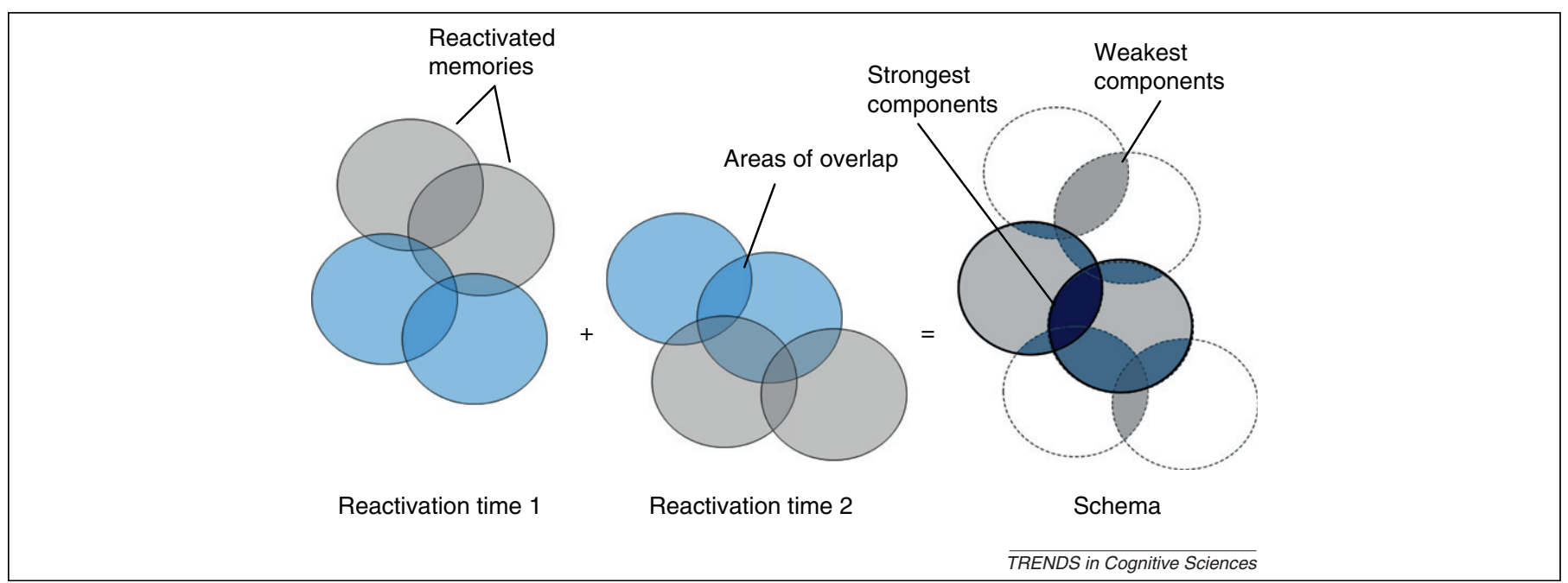

Figure 1. The iOtA concept. This diagram provides a schematic illustration of how strengthening memories that overlap during replay could lead to schema formation. Two sequential epochs of reactivation during the same sleep session are shown as Time 1 and Time 2. Circles represent replayed memories, with those in grey replayed during just one of the epochs shown and those in blue replayed during both. Note that overlap can occur either during simultaneous replay or via replay in more than one epoch (blue circles). The schema shown to the right of the equals sign is formed by a combination of replay, which strengthens areas of overlap, and global downscaling, which gradually erodes the more idyosncratic aspects of each memory. Within this schema, information that has been replayed more frequently and/or with more overlap will be more strongly represented. 


\section{Box 2. SWS and memory consolidation}

A prominent theory proposes that three characteristic field potentials, 'slow oscillations', 'spindles', and 'sharp wave ripples', are involved in some forms of sleep-dependent consolidation [11,14,43,58]. Adopting the widely used two-stage model of memory consolidation in which encoding first takes place within a fast hippocampal store (black arrow in Figure I) and is subsequently transferred offline to the neocortex over time (red arrow in Figure I) [17,34], this theory argues that slow oscillations play an essential role in coordinating this transfer.

'Slow oscillations', which arise in the neocortex but spread to many other areas [59] including the hippocampus and thalamus, and which occupy the $0.5-1.0 \mathrm{~Hz}$ band in electroencephalographic recordings, represent the synchronised membrane potential oscillations of millions of neurons. These oscillations provide an important temporal gating function because they cause widespread hyperpolarisation (inhibiting neural firing) during their 'down' state, and correspondingly widespread depolarisation (facilitating neural firing) during their 'up' state. During this up state, much faster and lower amplitude oscillations in the $11-15 \mathrm{~Hz}$ band called sleep 'spindle's occur in the thalamus and travel to neocortical areas [60]. These thalamocortical spindles stimulate early gene expression and glutamate receptors [43] thereby creating optimal conditions for long term potentiation (LTP) in the neocortex. At the same time, high frequency 'ripples' at
$100-300 \mathrm{~Hz}$ sitting on top of infrequent $50-100$ msec 'sharp waves' occur in the hippocampus. These are associated with the offline replay of neural sequences learned during encoding (Box 3). The temporal synchronisation of thalamocortical spindles and sharp waves by slow oscillations is believed to ensure that sequence replay in the hippocampus coincides with neocortical readiness for encoding, and thereby facilitates the transfer of sequences from the hippocampus to the neocortex $[14,43]$. This provides a potential physiological mechanism for offline consolidation of hippocampalbased memories during sleep $[2,61,62]$ (for reviews $[14,43]$ ).

SWS might also play a second complementary role in consolidation. If left unchecked, potentiation during sleep-dependent consolidation, as well as during wakeful encoding, would eventually lead to synaptic saturation. The synaptic homeostasis hypothesis $[29,30]$ proposes that all synapses belonging to neurons that were potentiated during the day are downscaled during SWS. Because each synapse is downscaled by a percentage of its current strength, the ratio between synapses is maintained. The theory also posits a threshold below which synapses will disappear altogether that could help to improve the signal-to-noise ratio of neuronal firing. Several authors have suggested that synaptic homeostasis and synaptic consolidation might work in concert during SWS $[11,12]$.

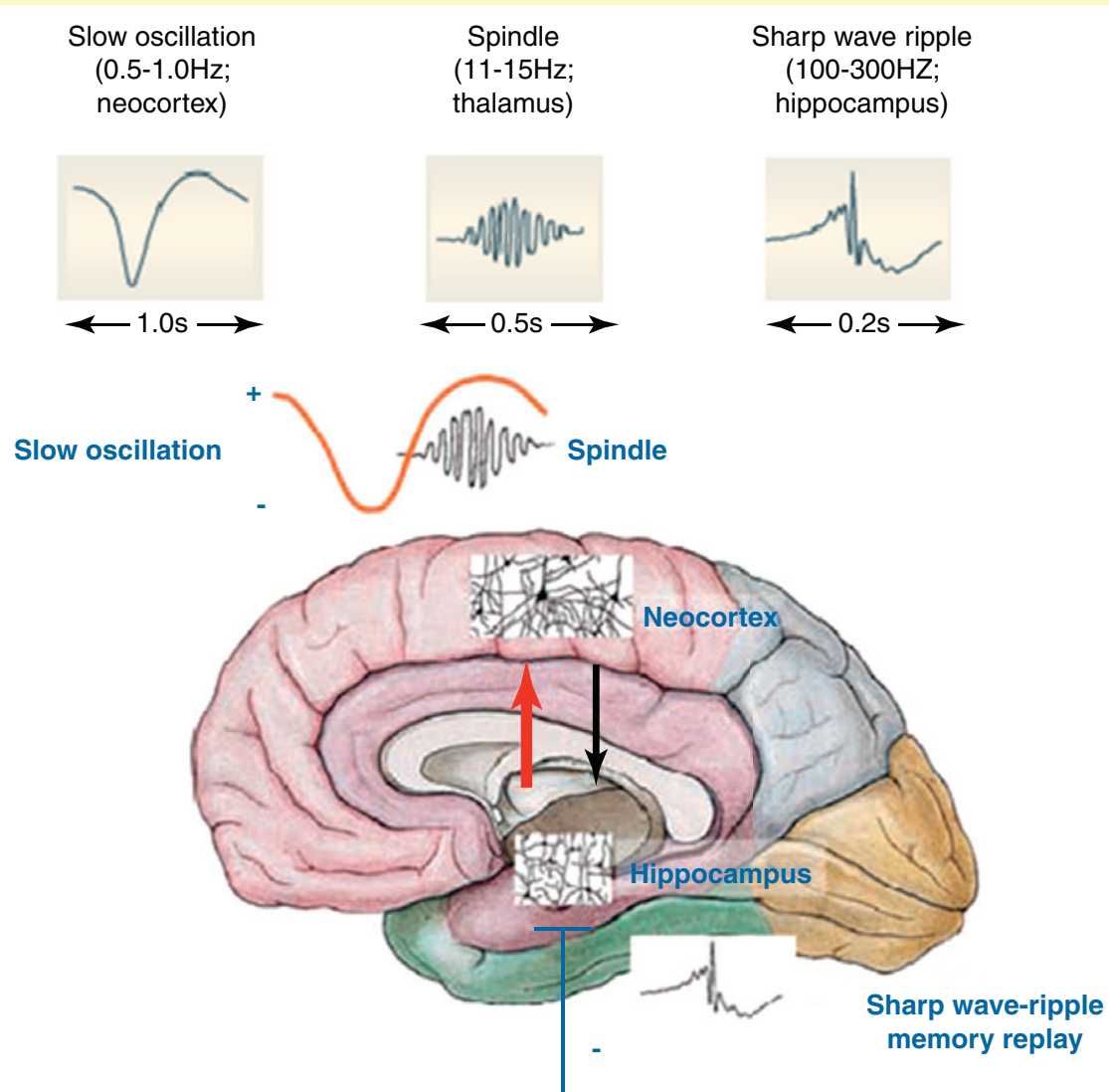

Acetylcholine, cortisol, etc.

Figure I. Consolidation during SWS. Adapted, with permission from [11] and [14]. The model (bottom) posits that the slow oscillations (top left) coordinate LTPenabling spindles (top centre) and replay-associated sharp-wave ripples (top right) to allow transfer from the hippocampus to the neocortex.

explain how, in combination with the synaptic downscaling that occurs during SWS [29,30], such overlap could lead to the gradual formation of conceptual schemata.

Overlap in memory reactivation can occur either simultaneously or sequentially (Figure 1). If simultaneous, neurons that code for shared memory components will be more strongly activated as manifest by increased firing rates. Where these neurons share synaptic interconnections, such enhanced firing can facilitate Hebbian learning. Although this learning will initially strengthen all interconnections between two simultaneously replayed memories, areas that are associated with both memories 
Reactivation of memory traces is an important mechanism for offline consolidation. Hippocampal 'place' cells fire only when a rat is in a specific location in a known environment. This means that cells fire in an ordered sequence when the rat moves along a particular route (see Figure I). Early work by Wilson and McNaughton [39] showed that cells that cofire during learning are more likely to cofire again during SWS. More recent work by Lee and Wilson [40] demonstrates that a series of place cells that fires during learning fires again in the same order during SWS, neuronally 'replaying' the rat's earlier movement but now speeded up 6-20 times. Importantly, this replay seems to coincide with the sharp wave ripples that originate in the hippocampus and are characteristic of SWS. These have been shown to be important for spatial learning in rats, with disruption of sharp wave ripples resulting in severely impaired consolidation $[63,64]$, Figure I.

During wakeful resting, rats also show replay but of a more exploratory nature. Replay in this context can reverse a firing sequence, starting at the current location and moving backwards through time [65]. Wakeful rats can even 'replay' novel routes that have not actually been experienced [66].

Identifying neuronal replay in humans is difficult due to the technical limitations involved but there is converging evidence that human reactivation occurs during both sleep and wakefulness. Reactivation of the hippocampus was observed during sleep following a spatial navigation task [67], whereas visual texture discrimination led to reactivation in those parts of $\mathrm{V} 1$ that are specifically involved in learning the task [23]. Importantly, the level of reactivation predicted subsequent performance improvement in both of these tasks.

Active stimulation of reactivation during sleep has provided additional evidence for a causal relation between reactivation and memory consolidation. For instance, re-presenting an odour that was present during encoding during subsequent sleep led to improved performance on a spatial memory task [24]. Notably, this improvement only occurred when the odour was presented during SWS rather than wake or any other sleep stage. A similar improvement was seen in a space-object location task when semantically-related sounds associated with the object during encoding were re-presented during sleep [25]. In addition, electroencephalogram (EEG) measurement during sleep revealed that greater neural response to auditory cues during SWS was associated with greater improvement in postsleep performance. In combination with the rodent studies, these results suggest that reactivation is important for consolidation and requires

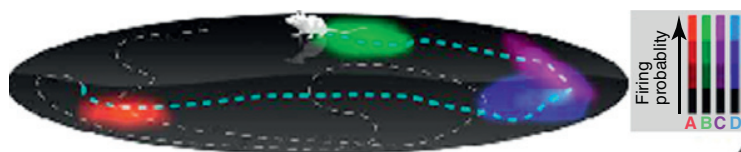

Exploration 1

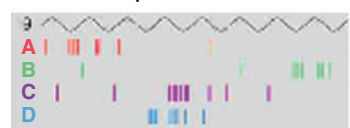

Sleep after

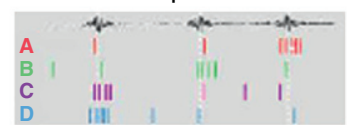

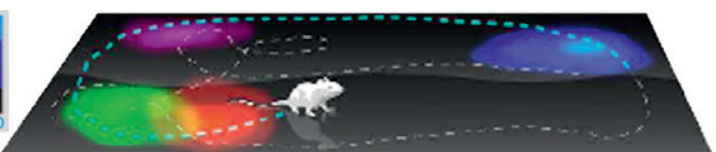

Exploration 2

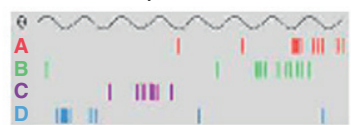

Sleep after

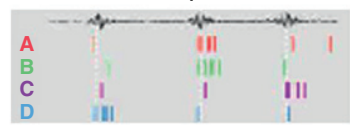

TRENDS in Cognitive Sciences

Figure I. Replay of neural sequences during sleep. Note that in the circular environment, locations $C$ and $D$ are close together, and place cells $C$ and $D$ subsequently fire simultaneously during sleep. Similarly, locations A and B are close together in the rectangular environment and again show simultaneous firing during subsequent sleep. Reproduced, with permission, from [68].

will be more strongly activated and their interconnections will therefore become stronger than connections relating to a single memory (Figure 2) [31]. Under the synaptic homeostasis hypothesis [29,30], recently potentiated synapses will be downscaled during SWS but the strongly potentiated interconnections between areas of overlap will survive this process. Conversely, Weaker connections between nonoverlapping portions of these memories will be lost during downscaling (Figure 2c). memory representations that have not been activated during the day preceding sleep (e.g. inactive memories) will not be influenced by this process because they will not be downscaled.

When overlapping memories are reactivated sequentially, neurons associated with multiple memories will again be more strongly activated than neurons associated with only one memory. Potentiation from the second reactivation will build upon remaining potentiation from the first, and so on. As with simultaneous reactivation, the resultant hyperpotentiation will lead to strong connections that have a higher chance of surviving subsequent downscaling. Connections that do survive will form a representation of the commonalities between memories that were reactivated during the night (Figure 2c). Sequential reactivation could also allow a subtle modulation of connection strengths that is not afforded by simultaneous reactivation. Downscaling occurs throughout SWS, especially in the early part of the night [32,33], so connections may begin to be downscaled in between widely spaced sequential reactivations. The extent of this downscaling will help to determine the extent to which areas of overlap are strengthened, and might represent one way in which connection strengths in the growing representation of overlap can be controlled. The number of times a memory is reactivated will also influence these connections and may therefore provide a second method for such control.

We call the general mechanism outlined above 'information overlap to abstract' or iOtA. As a means of consolidation, iOtA provides a powerful tool for the abstraction of shared elements in a set of related memories, for example the presents and cake that contribute to the general concept of a birthday party. Under iOtA, multiple bouts of reactivation in different combinations could act synergistically with downscaling to allow abstracted networks of connected neural nodes to gradually develop into a more detailed or accurate representation of the relation between various experiences, eventually producing what we call a schema. This selective strengthening by overlap could provide an efficient mechanism for schema formation during SWS. Importantly, although we have proposed potentiation as a result of replay, a highly localized reduction in downscaling would lead to identical results in terms of relative memory strength. Reduced downscaling as a result of replay should therefore be considered as an 
(a)

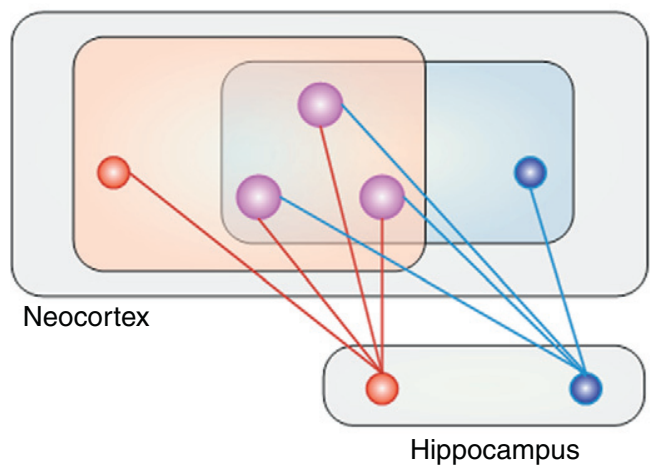

(b)

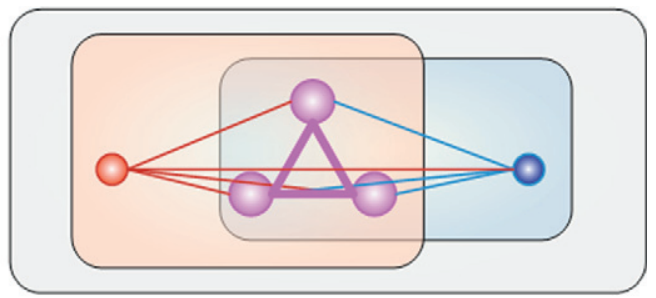

Neocortex

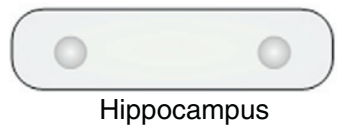

Synaptic downscaling

(c)

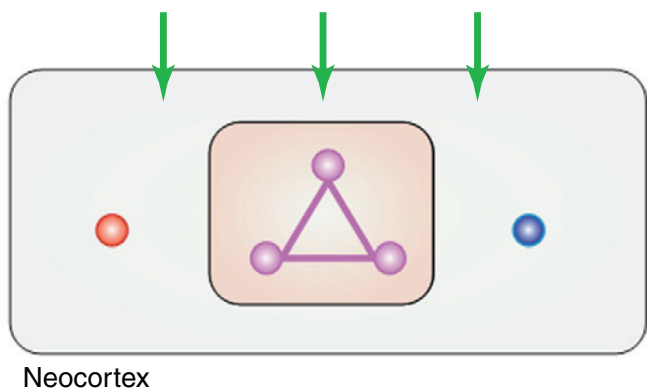

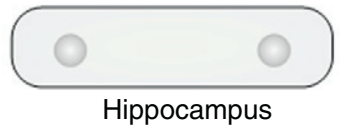

TRENDS in Cognitive Sciences

Figure 2. The iOtA mechanism. (a) Two memories are reactivated simultaneously. This reactivation, triggered by the hippocampus, includes some neocortical neurons unique to each memory, and some that are shared. (b) Shared neurons are potentiated more strongly and as a result develop stronger connections to each other than to neurons not involved in the overlap. (c) Following synaptic downscaling, only these strong connections between the shared neurons remain intact. This set of neurons now represents abstraction from the two memories, encoding their shared information only. In the figure, a larger neuron size indicates greater neural activation and a thicker line indicates a stronger synaptic connection.

alternate mechanism for abstraction under the iOtA framework.

Although there are strong parallels between the iOtA concept and prior models suggesting that episodic memories are gradually semanticised as they replay in SWS $[17,34]$, it is important to note that iOtA deals specifically with schema formation and makes no assumptions about semanticisation. The memories that are replayed in iOtA could be episodic but this is not essential because replay of overlapping memory fragments would also lead to abstraction. Similarly, the cognitive schemata formed by overlapping replay could represent a basic form of semantic knowledge but iOtA does not assume this.

\section{Adding to an existing schema}

Elegant work with rats suggests that memories that conform to an existing schema are assimilated rapidly into the neocortex, becoming independent of hippocampal connections much more quickly than those that do not [18]. The Complementary Learning Systems (CLS) model suggests that the interleaved reactivation of new and old memories during consolidation could be crucial for the integration of new memories into older neocortical traces [17]. iOtA builds on this idea to provide a more explicit mechanism for the integration of new memories by assuming that such reactivation triggers responses in schemata that are linked to the replayed memory via associative synapses. Such triggering entails activation of neurons in the schema that do not directly code for the new memory but that are part of the related schema (Figure 3). Activity in these additional schema-related neurons subsequently leads to an amplification of responses in the neocortical neurons involved in the new memory, and Hebbian learning then binds these neurons into the neocortical schema representation. Thus, newly learned information, and the existing schemata with which it overlaps, together form a positive reinforcement loop by which the new memory is both strengthened and incorporated into the schema.

Importantly, the linkages formed between a new memory and a schema during encoding and initial reactivation are unlikely to be strong enough to ensure that this memory will be triggered when the schema is next activated. Instead, these weak connections will be progressively downscaled and might eventually be lost. If a memory overlaps with the schema on multiple occasions, however, these connections will eventually become so strong that activity in the schema triggers responses in the 'new' memory (Figure 3h). This memory has been integrated into the schema. The requirement for multiple reactivations in conjunction with the schema is important because it means that only memories that are repeatedly associated with an existing schema will become integrated, biasing schemata towards incorporation of the shared aspects of a memory set and protecting them against incorporation of 


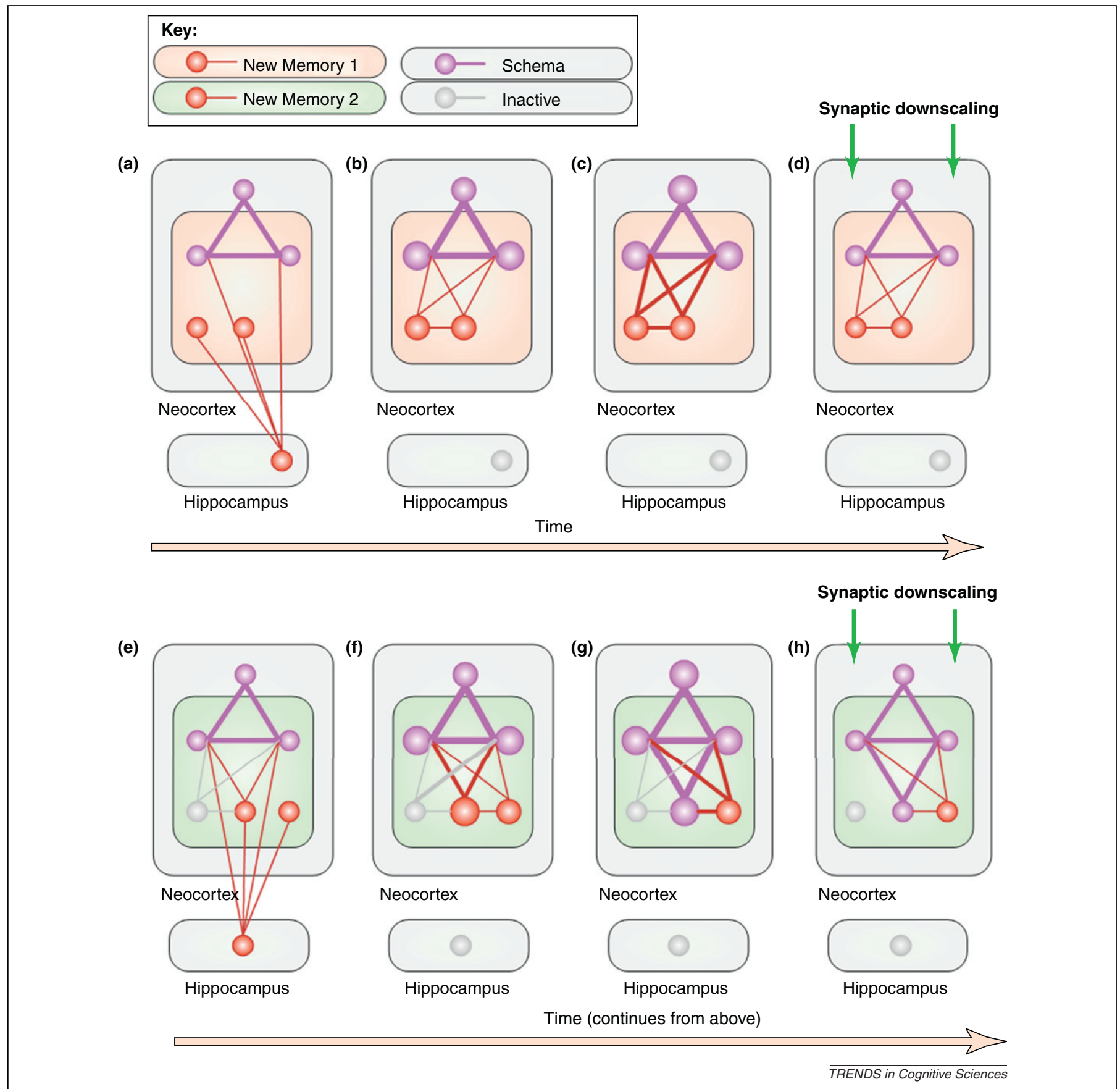

Figure 3. The assimilation of new memories into a schema. (a) A recently encoded memory, which shares some features with an existing schema, is reactivated. (b) This triggers the schema such that all additional neurons that are part of the schema become strongly activated. This, in turn, increases activation of all connected nonschematic

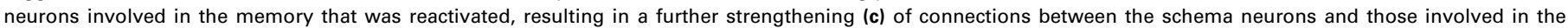
reactivated memory via Hebbian learning. (d) Synaptic downscaling brings the internal schema connections back to normal levels, and weakens the new connections. At this point, the new connections are not strong enough to allow activation of the schema alone to trigger activation of the new memory neurons; reactivation is still required. (e) A second recently encoded memory, which shares one nonschema neuron with the first new memory, is subsequently reactivated. (f) It follows the same process of

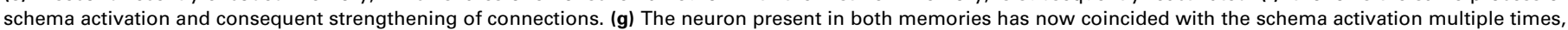
resulting in a connection strong enough to allow it to be activated by schema activation alone. (h) Synaptic downscaling removes the weak residual connections from the

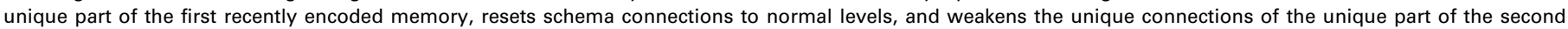
recently encoded memory such that it is not yet integrated into the schema.

less relevant information. Thus, if I attend several birthday parties in Mexico, where such events frequently involve a highly decorated box of sweets on a string called a piñata, the concept of the piñata will eventually become integrated into my birthday schema. By contrast, idiosyncratic details of the parties attended will not integrate because their association with the schema will be too weak to trigger activity in schema-related neurons.
The assimilation of new memories into a schema is modulated by the number of replays, the degree of overlap with the schema and the initial memory strength. Specifically, assimilation is more rapid and efficient, requiring a smaller number of replays, if there is extensive overlap between the new memory and the schema because few neurons in the new memory will need to be freshly interlinked. It is less efficient if overlap is limited because many 
new connections will need to be formed before the memory can become integrated. A weak memory with limited overlap could decay completely before it has been replayed often enough to integrate. If the same memory overlaps extensively it will integrate more quickly and will have a greater chance of being retained. Conversely, very strong memories can integrate into schemata with which they overlap only minimally at the outset because they are unlikely to decay before new associative linkages have been formed. This explains why schemata can be disproportionately influenced by especially strong memories [35-37].

\section{Integration, insight and false memories}

Recent work has demonstrated a role for sleep in improved integration [6-8], insight [9], and false memory formation [28]. iOtA provides a common mechanism for all of these effects. In one integration paradigm[6], participants implicitly learned a hierarchy of abstract images with the structure $\mathrm{A}>\mathrm{B}>\mathrm{C}>\mathrm{D}>\mathrm{E}>\mathrm{F}$. During training with feedback they were shown adjacent images from this hierarchy (e.g. B/C and C/D) and they were asked to indicate which was higher $(>)$. After consolidation, they were shown image pairs that were distant in the hierarchy (e.g. B/D or $\mathrm{B} / \mathrm{F}$ ), such that correct responses required integration of encoded pairs (e.g. $\mathrm{B}>\mathrm{C}+\mathrm{C}>\mathrm{D}=\mathrm{B}>\mathrm{C}>\mathrm{D}$ ). Importantly, this task was performed better after sleep. The insight paradigm [9] also required integration. Participants performed a 'number reduction task' in which a series of interlinked rote calculations produced a final cumulative answer. Participants who gained insight noticed an underlying pattern in the task such that the answer to the second calculation was also the final answer they sought. Similar to the integration task, the insight task was performed better after sleep. iOtA predicts that overlapping replay of learned information (training pairs in the integration task, and problems completed in the insight task) constructs an abstracted representation that should facilitate correct responding. In one case replay allows construction of the full hierarchy of learned stimuli, and in the other it facilitates realisation of the hidden rule regarding repetition of answers.

A recent study of false memory formation [28] followed similar principles but with additional reliance on existing associations or minischemata. Participants encoded a list of semantically related words such as 'cake, candles, presents and party' but not the gist word 'birthday'. Because the gist word is associated with each of the learned words, however, the sequential activation of the learned words at encoding led to preferential strengthening of the gist word representation and the binding of this word to each learned word. During subsequent free recall, experimenters kept track of both correct memories and erroneously reported gist words. The observed increase in false memories after sleep can be explained by iOtA because, under our model, reactivation of learned words during SWS would stimulate activity in the neural representation of associated gist words. The links between gist words and learned words would therefore be strengthened across sleep such that gist words are more strongly primed by cue words, increasing the chance that participants will remember the gist and produce a false memory.

\section{Why sleep?}

Is sleep necessary for abstraction? In considering this issue we should note that the behavioural studies of integration [6-8], abstraction [1-5], insight [9] and false memory formation [28] discussed above all suggest that sleep facilitates the formation of connections between related items. Importantly, performance on these tasks typically improves across both wakefulness and sleep but improvement is greater across the latter. This pattern is particularly apparent in our own study of statistical learning [38] that shows that time and sleep independently facilitate abstraction of the statistical properties underpinning a set of learned stimuli. Importantly, this facilitation was significantly greater across sleep and was predicted by slow wave activity. Such data suggest that the connections in question can be forged during wakefulness but that this process is facilitated by SWS. In iOtA we propose that this facilitated abstraction results from a unique combination of factors that is specific to SWS. First, the veridical replay of newly learned memories during this sleep stage $[39,40]$ (Box 3) is crucial to our model. Second, the targeted synaptic downscaling of only those neurons that were active during the previous period of wakefulness $[30,32,33,41]$ is essential. Third, the comparatively low level of sensory input experienced during SWS is an important way of minimising interference from external sources. Finally, the drastic reduction in acetylcholine that occurs during SWS, and that is essential for the transfer of information from hippocampal to neocortical memory stores [42], could also facilitate abstraction. Our proposal that SWS facilitates abstraction builds upon several influential theories of consolidation $[14,17,34,43]$ which argue that slow waves assist the transfer of hippocampal memories to the neocortex (Box 2). It also builds upon prior suggestions that SWS-based transfer of information might combine with synaptic downscaling to erase memories in which connections have not been sufficiently strengthened by replay $[12,30,44]$. In sum, although there is ample evidence that abstraction can occur during wake, this process is facilitated by sleep [1-5]. We argue that the unique combination of veridical replay, synaptic downscaling, comparative sensory isolation and favourable neuropharmacology that occurs during SWS could underpin this facilitation.

In addition to SWS, rapid eye movement (REM) sleep could also play a role in memory consolidation. There is evidence for a role of REM in the selective consolidation of emotional memories $[45,46]$ and in consolidation of procedural memory $[47,48]$. There is also limited evidence for involvement of REM in integration or abstraction [10,47] particularly when experienced synergistically with SWS [49]. Furthermore, connectionist modelling has suggested an elegant mechanism by which REM could strengthen weak memories [50]. Because there is limited evidence for reactivation of recently learned memories during REM, however (but see [47,51]), it seems unlikely that this sleep stage could contribute to the type of abstraction that we address in iOtA. Instead, REM might be crucial for other types of consolidation, and if it plays a role in abstraction, then the mechanism for this is likely to be distinct from that which occurs during SWS. 


\section{Box 4. Questions for future research}

- Are stronger or more emotional memories replayed more often during SWS?

- How is abstraction accomplished during wake?

- How does each of the following contribute to the construction of a schema: the strength of original memories, the number of reactivations, and the amount of downscaling that occurs between these?

- How does abstraction evolve across multiple nights? Do processes in sleep and wake build upon each other?

- How is LTP related to systems consolidation during SWS?

- Can LTP (due to replay) and long-term depression (due to downscaling) both be induced during SWS?

- Could reactivation lead to a temporary reduction in downscaling?

- What role, if any, does REM play in abstraction?

\section{Concluding remarks}

This article has introduced iOtA, a physiologically plausible mechanism by which the overlapping replay of newly learned memories during SWS could lead to the abstraction of key shared components or 'gist', and thus to the formation of new cognitive schemata. iOtA also provides a potential mechanism for the integration of newly learned information into existing schemata. We discuss this mechanism in the context of consolidation during SWS, and have shown how it can explain the greater integration, insight, and false memory formation that occur after SWS. It is important to note, however, that iOtA is concerned exclusively with abstraction and integration rather than with memory consolidation more widely. We therefore expect this mechanism to work in parallel with other forms of consolidation.

Our proposal is theoretical at present but future research into sleep-dependent abstraction, schema formation, replay, and their influence on memory, will determine its validity (see also Box 4 for key questions for future research). In rats, such work could look for replay of overlapping neuronal sequences and determine whether these are better remembered than nonoverlapping sequences. In humans, similar studies could draw on the recently demonstrated ability of sounds [25] and odours [24,26], which are paired with learned information at encoding and then re-presented during SWS, to trigger reactivation. This strategy would make it possible to directly engineer replay of overlapping memories during SWS, and to test the importance of such overlap for subsequent memory strength. Assuming these studies show that overlapping memory components are more strongly consolidated by replay than idiosyncratic components, related paradigms might later test the value of triggered replay for engineering the formation of more complex schemata. If successful, this 'schema engineering' could be used as a targeted learning technique in future. This strategy might also prove valuable for clinical applications where schema therapy [36] can help to combat depression, anxiety or chronically low self-esteem.

\section{Acknowledgements}

We thank Atsuko Takashima, Michael Zugaro, Jakke Tamminen, Susanne Diekelmann, Ken Norman, and three anonymous reviewers for helpful comments on the manuscript. We also thank Matt Wilson for helpful discussion of the ideas presented here. This work was funded by a BBSRC New Investigator Award [BB/F003048/1] to PL.

\section{References}

1 Gomez, R.L. et al. (2006) Naps promote abstraction in languagelearning infants. Psychol. Sci. 17, 670-674

2 Hupbach, A. et al. (2009) Nap-dependent learning in infants. Dev. Sci. 12, 1007-1012

3 Fischer, S. et al. (2006) Implicit learning-explicit knowing: a role for sleep in memory system interaction. J. Cogn. Neurosci. 18, 311-319

4 Durrant, S. and Lewis, P.A. (2009) Memory consolidation: tracking transfer with functional connectivity. Curr. Biol. 19, R860-R862

5 Djonlagic, I. et al. (2009) Sleep enhances category learning. Learn. Mem. 16, 751-755

6 Ellenbogen, J.M. et al. (2007) Human relational memory requires time and sleep. Proc. Natl. Acad. Sci. U.S.A. 104, 7723-7728

7 Tamminen, J. et al. (2010) Sleep spindle activity is associated with the integration of new memories and existing knowledge. J. Neurosci. 30 , $14356-14360$

8 Dumay, N. and Gaskell, M.G. (2007) Sleep-associated changes in the mental representation of spoken words. Psychol. Sci. 18, 35-39

9 Wagner, U. et al. (2004) Sleep inspires insight. Nature 427, 352-355

10 Cai, D.J. et al. (2009) REM, not incubation, improves creativity by priming associative networks. Proc. Natl. Acad. Sci. U.S.A. 106, 1013010134

11 Diekelmann, S. and Born, J. (2010) The memory function of sleep. Nat. Rev. Neurosci. 11, 114-126

12 Walker, M.P. (2009) The role of sleep in cognition and emotion. Ann. N. Y. Acad. Sci. 1156, 168-197

13 Stickgold, R. (2005) Sleep-dependent memory consolidation. Nature 437, 1272-1278

14 Born, J. (2010) Slow-wave sleep and the consolidation of long-term memory. World J. Biol. Psychiatry 11 (Suppl. 1), 16-21

15 Buzsaki, G. (1989) Two-stage model of memory trace formation: a role for "noisy" brain states. Neuroscience 31, 551-570

16 Marr, D. (1971) Simple memory: a theory for archicortex. Philos. Trans. R. Soc. Lond. B: Biol. Sci. 262, 23-81

17 McClelland, J.L. et al. (1995) Why there are complementary learning systems in the hippocampus and neocortex: insights from the successes and failures of connectionist models of learning and memory. Psychol. Rev. 102, 419-457

18 Tse, D. et al. (2007) Schemas and memory consolidation. Science 316, $76-82$

19 van Kesteren, M.T. et al. (2010) Persistent schema-dependent hippocampal-neocortical connectivity during memory encoding and postencoding rest in humans. Proc. Natl. Acad. Sci. U.S.A. 107, $7550-7555$

20 van Kesteren, M.T. et al. (2010) Retrieval of associative information congruent with prior knowledge is related to increased medial prefrontal activity and connectivity. J. Neurosci. 30, 15888-15894

21 Peyrache, A. et al. (2009) Replay of rule-learning related neural patterns in the prefrontal cortex during sleep. Nat. Neurosci. 12 919-926

22 Euston, D.R. et al. (2007) Fast-forward playback of recent memory sequences in prefrontal cortex during sleep. Science 318, 11471150

23 Yotsumoto, Y. et al. (2009) Location-specific cortical activation changes during sleep after training for perceptual learning. Curr. Biol. 19, 1278-1282

24 Rasch, B. et al. (2007) Odor cues during slow-wave sleep prompt declarative memory consolidation. Science 315, 1426-1429

25 Rudoy, J.D. et al. (2009) Strengthening individual memories by reactivating them during sleep. Science 326,1079

26 Diekelmann, S. et al. (2011) Labile or stable: opposing consequences for memory when reactivated during waking and sleep. Nat. Neurosci. 14 , 381-386

27 Kumaran, D. et al. (2009) Tracking the emergence of conceptual knowledge during human decision making. Neuron 63, 889-901

28 Diekelmann, S. et al. (2010) Sleep enhances false memories depending on general memory performance. Behav. Brain Res. 208, 425-429

29 Tononi, G. and Cirelli, C. (2003) Sleep and synaptic homeostasis: a hypothesis. Brain Res. Bull. 62, 143-150

30 Tononi, G. and Cirelli, C. (2006) Sleep function and synaptic homeostasis. Sleep Med. Rev. 10, 49-62

31 Abbott, L.F. and Nelson, S.B. (2000) Synaptic plasticity: taming the beast. Nat. Neurosci. 3 (Suppl.), 1178-1183

32 Huber, R. et al. (2004) Local sleep and learning. Nature 430, 78-81 
33 Tononi, G. (2009) Slow wave homeostasis and synaptic plasticity. J. Clin. Sleep Med. 5, S16-S19

34 Frankland, P.W. and Bontempi, B. (2005) The organization of recent and remote memories. Nat. Rev. Neurosci. 6, 119-130

35 Waring, J.D. and Kensinger, E.A. (2011) How emotion leads to selective memory: neuroimaging evidence. Neuropsychologia 49, 1831-1842

36 Schmidt, N.B. et al. (1995) The schema questionnaire: investigation of psychometric properties and the hierarchical structure of a measure of maladaptive schemas. Cogn. Ther. Res. 19, 295-321

37 Young, J.E. (1990) Cognitive Therapy for Personality Disorders: A Schema-focused Approach, Professional Resource Exchange

38 Durrant, S.J. et al. (2011) Sleep-dependent consolidation of statistical learning. Neuropsychologia 49, 1322-1331

39 Wilson, M.A. and McNaughton, B.L. (1994) Reactivation of hippocampal ensemble memories during sleep. Science 265, 676-679

40 Lee, A.K. and Wilson, M.A. (2002) Memory of sequential experience in the hippocampus during slow wave sleep. Neuron 36, 1183-1194

41 Huber, R. et al. (2006) Arm immobilization causes cortical plastic changes and locally decreases sleep slow wave activity. Nat. Neurosci. 9, 1169-1176

42 Gais, S. and Born, J. (2004) Low acetylcholine during slow-wave sleep is critical for declarative memory consolidation. Proc. Natl. Acad. Sci. U.S.A. 101, 2140-2144

43 Born, J. et al. (2006) Sleep to remember. Neuroscientist 12, 410-424

44 Walker, M.P. and van der, H.E. (2009) Overnight therapy? The role of sleep in emotional brain processing. Psychol. Bull. 135, 731-748

45 Nishida, M. et al. (2009) REM sleep, prefrontal theta, and the consolidation of human emotional memory. Cereb. Cortex 19, 11581166

46 Wagner, U. et al. (2001) Emotional memory formation is enhanced across sleep intervals with high amounts of rapid eye movement sleep. Learn. Mem. 8, 112-119

47 Maquet, P. et al. (2000) Experience-dependent changes in cerebral activation during human REM sleep. Nat. Neurosci. 3, 831-836

48 Stickgold, R. et al. (2000) Visual discrimination task improvement: a multi-step process occurring during sleep. J. Cogn Neurosci. 12, 246 254

49 Walker, M.P. and Stickgold, R. (2010) Overnight alchemy: sleepdependent memory evolution. Nat. Rev. Neurosci. 11, 218

50 Norman, K.A. et al. (2005) Methods for reducing interference in the Complementary Learning Systems model: oscillating inhibition and autonomous memory rehearsal. Neural Netw. 18, 1212-1228
51 Louie, K. and Wilson, M.A. (2001) Temporally structured replay of awake hippocampal ensemble activity during rapid eye movement sleep. Neuron 29, 145-156

52 Howard, R.W. (1987) Concepts and Schemata: An Introduction, Cassell

53 Rumelhart, D.E. (1980) Schemata: the building blocks of cognition. In Theoretical Issues in Reading Comprehension (Spiro, R.J. et al., eds), Erlbaum

54 Mandler, J.M. (1984) Stories, Scripts, and Scenes: Aspects of Schema Theory, L. Erlbaum Associates

55 Piaget, J. (1971) Biology and Knowledge, University of Chicago Press

56 Bartlett, F.C. (1932) Remembering: A Study in Experimental and Social Psychology, Cambridge University Press

57 Schmidt, R.A. (1975) A schema theory of discrete motor skill learning. Psychol. Rev. 82, 225-260

58 Steriade, M. and Buzsaki, G. (1990) Parallel activation of the thalamus and neocortex. In Brain Cholingeric Systems (Steriade, M. and Biesold, D., eds), pp. 3-64, Oxford University Press

59 Massimini, M. et al. (2004) The sleep slow oscillation as a traveling wave. J. Neurosci. 24, 6862-6870

60 Mander, B.A. et al. (2011) Wake deterioration and sleep restoration of human learning. Curr. Biol. 21, R183-R184

61 Siapas, A.G. and Wilson, M.A. (1998) Coordinated interactions between hippocampal ripples and cortical spindles during slow-wave sleep. Neuron 21, 1123-1128

62 Sirota, A. et al. (2003) Communication between neocortex and hippocampus during sleep in rodents. Proc. Natl. Acad. Sci. U.S.A. 100, 2065-2069

63 Ego-Stengel, V. and Wilson, M.A. (2010) Disruption of rippleassociated hippocampal activity during rest impairs spatial learning in the rat. Hippocampus 20, 1-10

64 Girardeau, G. et al. (2009) Selective suppression of hippocampal ripples impairs spatial memory. Nat. Neurosci. 12, 1222-1223

65 Foster, D.J. and Wilson, M.A. (2006) Reverse replay of behavioural sequences in hippocampal place cells during the awake state. Nature $440,680-683$

66 Gupta, A.S. et al. (2010) Hippocampal replay is not a simple function of experience. Neuron 65, 695-705

67 Peigneux, P. et al. (2003) Learned material content and acquisition level modulate cerebral reactivation during posttraining rapid-eyemovements sleep. Neuroimage 20, 125-134

68 O'Neill, J. et al. (2010) Play it again: reactivation of waking experience and memory. Trends Neurosci. 33, 220-229 